\title{
Do You Approach Positive Events or Do They Approach You? Linking Event Valence and Time Representations in a Dutch Sample
}

\author{
Annemijn C. Loermans \\ Assistant Professor in Marketing, Institute of Management Studies, \\ Goldsmiths, University of London, London, UK \\ a.loermans@gold.ac.uk
}

Bjorn B. de Koning

Associate Professor of Educational Psychology, Department of Psychology, Education, and Child Studies (DPECS), Erasmus School of Social and Behavioural Sciences, Erasmus University Rotterdam, Rotterdam, The Netherlands

b.b.dekoning@essb.eur.nl

\section{Lydia Krabbendam}

Full Professor Developmental Neuropsychology, Department of Clinical, Neuro- \& Developmental Psychology, Faculty of Behavioural and Movement Sciences, Vrije Universiteit Amsterdam, Amsterdam, The Netherlands lydia.krabbendam@vu.nl

\begin{abstract}
In order to think and talk about time, people often use the ego- or time-moving representation. In the ego-moving representation, the self travels through a temporal landscape, leaving past events behind and approaching future events; in the time-moving representation, the self is stationary and temporal events pass by. Several studies contest to the psychological ramifications of these two representations by, inter alia, demonstrating a link between them and event valence. These studies have, however, been limited to English speakers, even though language has been found to affect time representation. The present study therefore replicated Margolies and Crawford's (2008) experiment on event valence and time representation amongst speakers of Dutch. Unlike Margolies and Crawford (2008), we do not find that positive valence leads to the endorsement of an ego-moving statement. Future studies will need to determine
\end{abstract}


the ways through which language might moderate the relation between event valence and time representation.

\section{Keywords}

time representation - valence - ego-moving - time-moving - affective embodiment

Although being an omnipresent force in shaping daily life, time is an abstract concept that cannot be directly experienced through the senses. The cognitive representation of time thus poses a challenge to embodied accounts of cognition, which suppose that all cognitive representations are simulations of our sensory, motor, and introspective states (Barsalou, Niedenthal, Barbey, \& Ruppert, 2003; Wu \& Barsalou, 2009). Research addressing this challenge has found that, consistent with Conceptual Metaphor Theory, the concrete domain of space is often used to represent and talk about time (Boroditsky, 200o; Casasanto \& Boroditsky, 20o8; Lakoff \& Johnson, 1980; for a notable exception, see Sinha, Sinha, Zinken, \& Sampaio, 2011). Different spatial metaphors are used to understand and talk about time (for an overview see Bender \& Beller, 2014, or Núñez \& Cooperrider, 2013). Specific attention has been devoted to the ego-moving and time-moving representations (Boroditsky \& Ramscar, 2002; Duffy \& Feist, 2014; Duffy, Feist, \& McCarthy, 2014; Feist \& Duffy, 2015; Gentner, Imai, \& Boroditsky, 2002; Ruscher, 2011; for examples of other spatial metaphors, please see Boroditsky, Fuhrman, \& McCormick, 2011; Boroditsky \& Gaby, 2010; de la Fuente, Santiago, Roman, Dumitrache, \& Casasanto, 2014; Núñez, Cooperrider, Doan, \& Wassmann, 2012). With the ego-moving representation, people see themselves as moving through a temporal landscape, towards future events ahead, whilst leaving past events behind (e.g., We are approaching the deadline). With the time-moving representation, people construe time as moving and consider themselves as stationary, much like a river flows past stationary observers on a bank (e.g., The deadline is approaching).

A plethora of studies show that these two representations are psychologically meaningful in the sense that using one representation over the other is determined, in part, by one's personality and/or contextual factors (Boroditsky \& Ramscar, 2002; Duffy \& Feist, 2014; Duffy et al., 2014). Most relevant to the current study are the seminal studies by McGlone and Pfiester (2009) and Margolies and Crawford (2008) that indicate that the ego-moving representation is linked to positive valence while the time-moving representation is linked to negative valence. Margolies and Crawford (2008) found, in two 
experiments, that when participants were asked to think of a positive future event, as opposed to a negative future event, they were more likely to endorse the statement that they were approaching the event, as opposed to the statement that the event was approaching them. They also found that when reading a vignette with ego-moving expressions (e.g., As I got closer to the event ...), as opposed to time-moving expressions (e.g., As the event got closer ...), participants were more likely to infer that the person described in the vignette felt positive, rather than negative, about the event (Margolies \& Crawford, 2008). Similarly, McGlone and Pfiester (2009) analysed spoken and written English corpora and found that ego-moving expressions, as opposed to time-moving expressions, were more frequently used when describing a positive event, as opposed to a negative event. Moreover, when participants produced narratives about a positive event, as opposed to a negative event, they were more likely to employ egomoving expressions than time-moving expressions (McGlone \& Pfiester, 2009).

The above findings are grounded in research on the embodiment of affective experience: people tend to approach what is positive and avoid what is negative (Cacioppo, Priester, \& Berntson, 1993; Chen \& Bargh, 1999). As agency is assigned to the person in the ego-moving representation (the person moves towards temporal events), this representation is assumed to be preferred over the time-moving representation when talking about a positive event (Margolies \& Crawford, 2008; McGlone \& Pfiester, 2009). Corroborating evidence for the link between valence and the two time representations comes from studies investigating the link between ego- and time-moving representations and emotion. For example, across three experiments, Hauser, Carter, and Meier (2009) found that the ego-moving representation is linked to anger, providing evidence that the ego-moving representation is indeed grounded in an approach motivation. Similarly, Richmond, Wilson, and Zinken (2012) showed that an ego-moving representation is linked with higher agency, happiness, and a future temporal orientation, whilst a time-moving representation is linked with lower agency, depression, anxiety, and a fatalistic temporal orientation. Furthermore, a study by Lee and Ji (2014) revealed that recalling an unpleasant past event or a pleasant future event leads participants to adopt an ego-moving representation, whilst recalling a pleasant past event or an unpleasant future event leads participants to adopt a time-moving representation. Combined these studies provide evidence that agency is assigned to the person in the ego-moving representation, and that this representation is consequently grounded in an approach motivation, thereby linking the egomoving representation with positive event valence.

The studies by Margolies and Crawford (2008) and McGlone and Pfiester (2009) are restricted to English participants and English corpora, with no 
follow-up studies conducted to test the generalizability of their findings to non-English speakers or corpora. This is significant because cross-cultural research has shown that the use of the ego- and time-moving representations is strongly affected by language (Bender, Beller, \& Bennardo, 2010; Lai \& Boroditsky, 2013; Rothe-Wulf, Beller, \& Bender, 2015; for examples of crosscultural work where cultural factors other than language, like temporal orientation, are shown to affect time representation, please see de la Fuente, Santiago, Roman, Dumitrache, \& Casasanto, 2014; Li, Bui, \& Cao, 2018). For example, Lai and Boroditsky (2013) found that whilst Americans prefer the ego-moving representation, speakers of Mandarin prefer the time-moving representation (English-Mandarin bilinguals fall in-between; their preferred representation depends on the language in which they are asked). Additionally, Bender et al. (2010) and Rothe-Wulf et al. (2015) have shown that speakers of German, Swedish, and Tongan, in addition to speakers of English and Mandarin, also differ in their preferences for the ego- and time-moving representations. Given that such linguistic differences regarding the representation of time exist, there is a clear need for cross-linguistic replication studies linking the ego- and time-moving representations to event valence.

This study takes a first step in addressing this by testing the link between the ego- and time-moving representations and event valence amongst native Dutch speakers. Investigating this link among Dutch speakers enables us to extend prior work as Dutch speakers have been found to use both the ego- and time-moving representations (Elvevåg, Helsen, De Hert, Sweers, \& Storms, 2011). Specifically, we aimed to conceptually replicate Margolies and Crawford's (2008) second experiment, which manipulated event valence by providing participants with a positive and negative event. Participants given a positive event were more likely to endorse the ego-moving statement I am approaching the event than the timemoving statement The event is approaching me. It should be noted that no effect of event valance was found on answers to the ambiguous time question (Next week's Wednesday's meeting is moved forward by two days. On which day is the meeting now?), a measure widely used to gauge time representation (Boroditsky \& Ramscar, 2002; Lai \& Boroditsky, 2013; McGlone \& Harding, 1998; Rothe-Wulf et al., 2015). The answer to this question depends on which time representation people employ, with use of the ego-moving representation leading to a Friday answer and use of the time-moving representation leading to a Monday answer. We also included the ambiguous question in our set-up. We tested whether event valence affected time representation, specifically whether positive event valence made it more likely for Dutch participants to adopt an ego-moving representation and whether negative event valence made it more likely for Dutch participants to adopt a time-moving representation. 


\subsection{Participants and Design}

We recruited 197 participants for our study on the campus of Utrecht University, a sample size comparable to the sample size used in previous studies on this topic with a similar set-up (Margolies \& Crawford, 2008; McGlone \& Pfiester, 2009). We only included participants who indicated Dutch as their sole mother tongue for analyses, leaving us with a sample of 162 participants ( $79 \%$ female). Participants' age ranged between 17 and 67 years of age $\left(M=21.5^{\circ}\right.$, $\left.S D=5.61\right)$. They received a candy bar as token of appreciation for their participation. Participants were randomly assigned to one of three conditions that resulted from the between subjects-factor Event Valence (positive, neutral, negative).

\subsection{Materials and Procedure}

Materials and procedure were based on Margolies and Crawford (2008). In addition to the positive and negative event, we also included a neutral condition to explore which time representation is used by participants when thinking about a neutrally valenced event. All participants received a booklet in which they were first asked to imagine a positive event (a trip to their favourite place), a negative event (appointment with the dentist for a molar extraction), or a neutral event (an appointment), taking place next week Wednesday. Participants were asked to provide a brief description of the event.

To gauge time representation, we used the ambiguous Wednesday question (McGlone \& Harding, 1998) and Margolies and Crawford's (2008) question. For the ambiguous Wednesday question, participants were told the event was moved forward by two days and asked to indicate on what day the event would take place now that it had been rescheduled (with a Monday response being indicative of a time-moving representation and a Friday response being indicative of an ego-moving representation; Margolies \& Crawford, 2008; McGlone \& Harding, 1998). Margolies and Crawford's (2008) question measures time representation by asking participants to choose one of two statements, one phrased in an ego-moving manner (I am approaching the event) and one in a time-moving manner (The event is approaching me; Margolies \& Crawford, 2008). For half of the participants, the ego-moving statement was presented first, whereas for the other half the time-moving representation was presented first. Participants then indicated the degree to which they felt positive and enthusiastic about the event, the degree to which they dreaded the event, and how angry the event made them (Margolies \& Crawford, 2008). In contrast to Margolies and Crawford (2008), we counterbalanced the order of the time representation questions and questions regarding their positive and 
negative emotions to control for ordering effects. Lastly, participants indicated what they thought the research was about and provided demographic information. Participants were debriefed after their participation.

\section{$2 \quad$ Results}

One participant $(0.62 \%)$ was excluded from the analyses because this participant seemed aware of the purpose of the study. Three participants $(1.85 \%)$ were removed because they seemed aware of the ambiguous nature of the ambiguous time question. Fifteen participants (9.26\%) answered the ambiguous time question with a response other than Monday or Friday and were also excluded from the analyses. All analyses were performed on the data of the remaining 143 participants.

\subsection{Manipulation Check}

We first checked whether the events with positive, neutral, or negative valence successfully manipulated the feelings of participants as gauged by the emotion questions. The effect of event valence on the emotion questions was analysed with a series of one-way analyses of variance (ANOVAs) with planned comparisons. The order of time representation questions and the emotion questions did not significantly affect the answers to the emotion questions. Therefore, we collapsed across this factor in the analyses. As can be observed in Table 1, there was a significant effect of Condition on all emotion questions. As expected,

TABLE 1 Means, standard deviations, and analyses of variance on event valence and emotion questions

Event valence

Positive Neutral Negative

$\begin{array}{lllllllll}M & S D & M & S D & M & S D & F(2,140) & p & \eta^{2}\end{array}$

$\begin{array}{lccccccccc}\text { Positive feelings } & 6.24 \mathrm{a} & .80 & 4.17 \mathrm{~b} & 1.02 & 3.91_{\mathrm{b}} & 1.32 & 60.23 & <.001 & .462 \\ \text { Enthusiasm } & 6.41_{\mathrm{a}} & .77 & 3.76 \mathrm{~b} & 1.46 & 2.57 \mathrm{c} & 1.55 & 98.74 & <.001 & .585 \\ \text { Dread } & 1.66 \mathrm{a} & .97 & 3.72 \mathrm{~b} & 1.26 & 4.95 \mathrm{c} & 1.65 & 69.81 & <.001 & .499 \\ \text { Anger } & 1.27 \mathrm{a} & .74 & 2.61 \mathrm{~b} & 1.47 & 2.75 \mathrm{~b} & 1.56 & 16.38 & <.001 & .190\end{array}$

Note. Means with different subscripts within rows differ significantly at $p<$. oo1. 
the positive event invoked more positive feelings, more enthusiasm, less dread, and less anger than the neutral or negative event. The negative event invoked fewer positive feelings, less enthusiasm, more dread, and more anger than the neutral event, although these differences were only significant for enthusiasm and dread.

\subsection{Time Questions}

First, we analysed the answers to Margolies and Crawford's (2008) question. Seventy-three participants $(51 \%)$ chose the ego-moving statement (I am approaching the event); 67 participants $(46.9 \%)$ chose the time-moving statement (The event is approaching me); three participants ( $2.1 \%)$ did not answer. A chi-square analysis revealed a significant effect of the order of the time representation questions and the emotion questions on the question about whether the participants saw themselves as approaching the event or saw the event as approaching them, $\chi^{2}(1, N=140)=4.22, p=.044$ (please note that for all analyses in this paper, the chi-square value reported is the exact value and the $p$ value reported is two-sided). Of the participants who received the emotion questions first, a majority $(61.2 \%)$ chose the ego-moving statement, indicating that they saw themselves as approaching the event; in contrast, of the participants who received the time representation questions first, a majority $(56.2 \%)$ chose the time-moving statement, indicating that they saw the meeting as approaching them. So, to test for the effect of event valence on time representation, we performed a logistic regression analysis with event valence (positive, neutral, negative), order of time representation and emotion questions (time questions first, emotions questions first), and their interaction, as predictors. A test of the full model against a constant model was not statistically significant, $\chi^{2}(3, N=140)=4.424, p=.219$ (see Table 2). More specifically,

TABLE 2 Results of the logistic regression on Margolies and Crawford's (2008) question with event valence, order of time representation and emotion questions, and their interaction, as predictors

\begin{tabular}{llcccc}
\hline & & $B(S E)$ & Wald & $\exp B$ & \multicolumn{1}{c}{$p$} \\
\hline \multirow{2}{*}{ Included } & Constant & $-.66(.691)$ & .92 & .52 & .336 \\
& Event valence & $.10(.308)$ & .11 & 1.11 & .745 \\
& Order & $.74(.962)$ & .60 & 2.10 & .437 \\
& Event valence * Order & $-.02(.42)$ & .002 & .98 & .962 \\
\hline
\end{tabular}

Note. $R^{2}($ Cox \& Snell $)=.031, R^{2}($ Nagelkerke $)=.041$. 
participants who were thinking of a more positive event were not more likely to choose the ego-moving statement, indicating that they saw themselves as approaching the event, compared to participants who were thinking a neutral or positive event. There were no other significant main or interaction effects.

Next, we analysed the answers to the ambiguous time question. Almost all participants (93\%) gave a time-moving answer (Monday) in response to the ambiguous time question; only ten participants $(7 \%)$ gave an ego-moving answer (Friday). As expected counts were not above five for every cell, we conducted a Fisher's Exact Test to see whether event valence had an effect on answers to the ambiguous time question. We collapsed across order of time representation questions and emotion question, given that there were no significant order effects. This analysis showed that of the 133 participants providing a time-moving answer in response to the ambiguous time question, 39 $(29.3 \%)$ were in the positive event valence condition, $44(33.1 \%)$ were in the neutral event valence condition, and $50(37.6 \%)$ were in the negative event valence condition; these differences were not statistically significant, $p=.498$.

\subsection{Additional Analyses}

As indicated above, the results regarding event valence on Margolies and Crawford's (2008) question showed a non-significant effect in the direction opposite to the findings that have previously been reported for English speaking participants (Margolies \& Crawford, 2008). To quantify this non-significant finding, we followed Dienes' (2014) recommendation and performed an additional analysis using Bayesian statistics to report a Bayes Factor. Bayesian Contingency Tables Tests showed that for the effect of event valence on Margolies and Crawford's (2008) question, the $B F_{\mathrm{O} 1}$ was 5.954 , indicating that the data we observed were 5.954 more likely under the null hypothesis than the alternative hypothesis.

\section{Discussion}

This study tested whether the relation between positive event valence and the ego-moving representation, and conversely, negative event valence and the time-moving representation (Margolies \& Crawford, 2008; McGlone \& Pfiester, 2009) could also be observed amongst Dutch speakers. Our findings do not provide evidence in support of such a relation. Specifically, we could not replicate Margolies and Crawford's (2008) finding that thinking about a positive event, as opposed to a negative event, made it more likely for participants to endorse an ego-moving statement over a time-moving statement in native 
Dutch speakers. This contrasts with several previous studies, with English speakers and English corpora, that did report a link between event valence and time representation (Hauser et al., 2009; Margolies \& Crawford, 2008; McGlone \& Pfiester, 2009; Richmond et al., 2012). Additional studies might thus be warranted to test the robustness of the event valence effect and/or to determine whether this effect is moderated by language, to the extent that a possible robust effect in one language (e.g., English) does not necessarily generalize to other cultural and/or linguistic samples (e.g., Dutch).

The idea of cultural factors, like language, affecting time representation, is supported by previous research. For example, several studies have shown that cultural differences related to our writing system influence the direction in which we conceptualize the flow of time (Boroditsky et al., 2011; Tversky, Kugelmass, \& Winter, 1991). Similarly, de la Fuente, Santiago, Roman, Dumitrache, and Casasanto (2014) have shown that whether a culture emphasizes the past or future (i.e., temporal orientation), influences whether people place the future in front of them or behind them. Moreover, Lai and Boroditsky (2013) have argued that cognitive differences in relation to preferences for the ego-moving or time-moving representation in speakers of Mandarin and English are shaped by language, specifically the frequency of ego- and time-moving expressions in the two respective languages: Where both representations are readily observed in the English language, the timemoving representation is more frequent in Mandarin. This seems to affect the cognitive representation of time as speakers of Mandarin were observed to almost exclusively choose a time-moving consistent answer in response to ambiguous time questions (Lai \& Boroditsky, 2013). An interesting question for future research to explore is whether these differences in linguistic frequency are perhaps related to linguistic differences with regards to animacy (Gardelle \& Sorlin, 2018) or more general cultural differences with regards to anthropomorphism (Epley, Waytz, \& Cacioppo, 2007), in the sense that members of linguistic groups and cultures, which are more accepting of attributing animacy to inanimate objects (e.g., time), might employ the time-moving representation to think about time more frequently than members of linguistic groups and cultures which are not as accepting of attributing animacy to inanimate objects. More pertinent to the current research, however, is the question of whether the (possible) differences in linguistic frequency of the different time representations also affect how these representations are linked to psychological constructs such as event valence.

The idea of cultural differences vis-à-vis linguistic frequency affecting the way in which certain time representations are linked to psychological constructs is not unprecedented within the literature. Namely, a study by 
Rothe-Wulf et al. (2015) showed that the effect of spatial primes on the egomoving and time-moving representation of time, as reported in previous studies using English speaking participants (Boroditsky \& Ramscar, 2002; Casasanto \& Boroditsky, 2008), could not be observed amongst German and Swedish speakers. German and Swedish speakers showed a clear preference for the time-moving and ego-moving representations respectively, which diverges from the variation in answers observed amongst English speakers. Similarly, Loermans and Milfont (2018) could not replicate Richmond et al.'s (2012) findings regarding the relation between future orientation and time representation amongst New Zealand participants; New Zealand participants were reported to also have a slight preference for the time-moving representation, at least more so than other English speaking participants. This suggests that perhaps linguistic and/or cultural ambiguity vis-à-vis the ego-moving and time-moving representations, as is observed amongst certain groups of English speaking participants, is a prerequisite for these time representations to be differentially linked to such variables as event valence (and future orientation).

Our results provide conflicting indications on the Dutch preference for either the ego-moving or time-moving representation though: whilst findings on the ambiguous time question suggest a strong preference for the time-moving representation (almost all participants responded Monday), a large proportion of participants endorsed the ego-moving statement (I am approaching the event) over the time-moving statement (The event is approaching me) in Margolies and Crawford's (2008) question. This contrasts with research by Margolies and Crawford (2008) in which a similar proportion of ego- and time-moving responses across the two questions in the same population was observed. It also deviates from research by Richmond et al. (2012) who found that participants responding with an ego-moving answer to the ambiguous time question (Friday) tended to choose the ego-moving statement (I am approaching the event) more often. We interpret the large discrepancy between the two measures in our Dutch population as support for Margolies and Crawford's (2008) idea about how the two measures might tap into different constructs altogether. More importantly, however, it shows that future (replication) research investigating time representation (across cultures) and their psychological ramifications will need to carefully consider their methods and measures and (continue) to combine linguistic and cognitive approaches. Possibly certain measures (i.e., the ambiguous time questions) trigger a cultural and/or linguistic convention within some culture, and therefore are not suited to adequately capture the (variation) in the cognitive representation of time across the different cultures. 
An alternative explanation for our findings may be related to the role culture plays in shaping embodied processes (Cohen \& Leung, 20o9; Maass, 2009). Because cultures systematically vary with regards to which postures and comportments they encourage and discourage and vary in how they organize their physical space, certain embodied processes do not always generalize to other cultures. An example of this can be found in studies comparing the relation between honour and body comportment in honour and non-honour cultures (IJzerman \& Cohen, 2011). Perhaps this is also the case with regards to affective embodiment, specifically the idea that we (universally) approach what is good and avoid what is bad (Cacioppo et al., 1993; Chen \& Bargh, 1999) on which the hypothesized (universal) link between event valence and time representation is largely based. Namely, a recent study by Rotteveel et al. (2015) could not replicate the approach-avoidance finding reported by Chen and Bargh (1999). This may imply that this effect is not as robust as previously assumed, but it might also warrant investigation into whether this effect is perhaps somewhat culturally specific since Rotteveel et al. (2015) conducted their study amongst Dutch participants whilst Chen and Bargh (1999) used American participants.

Additionally, we found that participants who answered questions about how they felt, before answering time representation questions, were more likely to endorse an ego-moving statement (I am approaching the event) than a time-moving statement (The event is approaching me), compared to participants who answered the time representation questions before answering the time representation questions. This might be explained using the work by Gentsch and Synofzik (2014) who highlight the way emotions and affective experiences affect our sense of agency: perhaps the answering of introspective questions leads to increased feelings of agency. Increased feelings of agency lead, in turn, to the adoption of an ego-moving perspective (Richmond et al., 2012). Similarly, answering a question about how one feels is likely to put more focus on the self, which, in turn, might lead to the adoption of an ego-moving representation as this representation also highlights the self.

Concluding, using a Dutch sample, we did not replicate previous findings that a positive event leads to the adoption of an ego-moving representation (Margolies \& Crawford, 2008). This raises questions about the relation between event valence and the ego- and time-moving representations. Specifically, it highlights the need for future research to determine the robustness of the interplay between event valence and time representations, and the extent to which it generalizes across different languages. We believe that continued efforts towards replicating studies, with consideration of cultural factors, like 
language, as possible moderator of psychological processes, is pertinent for researchers in psychology trying to move the field forward.

\section{Funding}

This research was supported by a VICI grant from the Netherlands Organization for Scientific Research (NWo) awarded to Lydia Krabbendam (Grant No.453-11-0o5). The funders had no role in study design, data collection, and analysis, decision to publish, or preparation of the article.

\section{References}

Barsalou, L. W., Niedenthal, P. M., Barbey, A. K., \& Ruppert, J. A. (2003). Social embodiment. In B. H. Ross (Ed.), The Psychology of Learning and Motivation (pp. 43-92). San Diego: Academic Press.

Bender, A., \& Beller, S. (2014). Mapping spatial frames of reference onto time: A review of theoretical accounts and empirical findings. Cognition, 132(3), 342-382. http:// doi.org/10.1016/j.cognition.2014.03.016.

Bender, A., Beller, S., \& Bennardo, G. (2010). Temporal frames of reference: Conceptual analysis and empirical evidence from German, English, Mandarin Chinese and Tongan. Journal of Cognition and Culture, 10(3), 283-307. http://doi.org/ $10.1163 / 156853710 X_{531195}$.

Boroditsky, L. (2000). Metaphoric structuring: Understanding time through spatial metaphors. Cognition, 75(1), 1-28. http://doi.org/10.1016/Soo1o-0277(99)ooo73-6.

Boroditsky, L., Fuhrman, O., \& McCormick, K. (2011). Do English and Mandarin speakers think about time differently? Cognition, 118(1), 123-129. http://doi.org/10.1016/j .cognition.2010.09.010.

Boroditsky, L., \& Gaby, A. (2010). Remembrances of times east: Absolute spatial representations of time in an Australian aboriginal community. Psychological Science, 21(11), 1635-1639. http://doi.org/10.1177/o956797610386621.

Boroditsky, L., \& Ramscar, M. (2002). The roles of body and mind in abstract thought. Psychological Science, 13(2), 185-189. http://doi.org/10.1111/1467-9280.00434.

Cacioppo, J. T., Priester, J. R., \& Berntson, G. G. (1993). Rudimentary determinants of attitudes. II: Arm flexion and extension have differential effects on attitudes. Journal of Personality and Social Psychology, 65(1), 5-17. http://doi.org/ 10.1037/0022-3514.65.1.5.

Casasanto, D., \& Boroditsky, L. (2008). Time in the mind : Using space to think about time. Cognition, 106(2), 579-593. http://doi.org/10.1016/j.cognition.2007.03.004. 
Chen, M., \& Bargh, J. A. (1999). Consequences of automatic evaluation: Immediate behavioral predispositions to approach or avoid the stimulus. Personality and Social Psychology Bulletin, 25(2), 215-224. http://doi.org/10.1177/0146167299025002007.

Cohen, D., \& Leung, A. K.-Y. (2009). The hard embodiment of culture. European Journal of Social Psychology, 39(7), 1278-1289. http://doi.org/10.1002/ejsp.671.

de la Fuente, J., Santiago, J., Roman, A., Dumitrache, C., \& Casasanto, D. (2014). When you think about it, your past is in front of you: How culture shapes spatial conceptions of time. Psychological Science, 25(9), 1682-169o. http://doi.org/ 10.1177/0956797614534695.

Dienes, Z. (2014). Using Bayes to get the most out of non-significant results. Frontiers in Psychology, 5, 1-17. http://doi.org/10.3389/fpsyg.2014.00781.

Duffy, S. E., \& Feist, M. I. (2014). Individual differences in the interpretation of ambiguous statements about time. Cognitive Linguistics, 25(1), 29-54. http://doi.org/10.1515/ cog-2013-0O3O.

Duffy, S. E., Feist, M. I., \& McCarthy, S. (2014). Moving through time: The role of personality in three real-life contexts. Cognitive Science, 38, 1662-1674. http://doi .org/10.1111/cogs.12124.

Elvevåg, B., Helsen, K., De Hert, M., Sweers, K., \& Storms, G. (2011). Metaphor interpretation and use: A window into semantics in schizophrenia. Schizophrenia Research, 133, 205-211. http://doi.org/10.1016/j.schres.2011.07.oog.

Epley, N., Waytz, A., \& Cacioppo, J. T. (2007). On seeing human: A three-factor theory of anthropomorphism. Psychological Review, 114(4), 864-886. http://doi.org/ 10.1037/0033-295X.114.4.864.

Feist, M. I., \& Duffy, S. E. (2015). Moving beyond "Next Wednesday": The interplay of lexical semantics and constructional meaning in an ambiguous metaphoric statement. Cognitive Linguistics, 26(4), 633-656. http://doi.org/10.1515/cog-2015-0052.

Gardelle, L., \& Sorlin, S. (2018). Anthropocentrism, egocentrism and the notion of Animacy Hierarchy. International Journal of Language and Culture, 5(2), 133-162. http://doi.org/10.1075/ijolc.oooo4.gar.

Gentner, D., Imai, M., \& Boroditsky, L. (2002). As time goes by: Evidence for two systems in processing space $\rightarrow$ time metaphors. Language and Cognitive Processes, $17(5)$, 537-565. http://doi.org/10.1080/o169o96o1430oo317.

Gentsch, A., \& Synofzik, M. (2014). Affective coding: the emotional dimension of agency. Frontiers in Human Neuroscience, 8, 1-7. http://doi.org/10.3389/fnhum.2015.00142.

Hauser, D. J., Carter, M. S., \& Meier, B. P. (2009). Mellow Monday and furious Friday: The approach-related link between anger and time representation. Cognition \& Emotion, 23(6), 1166-118o. http://doi.org/10.108o/o2699930802358424.

IJzerman, H., \& Cohen, D. (2011). Grounding cultural syndromes: Body comportment and values in honor and dignity cultures. European Journal of Social Psychology, 41, 456-467. http://doi.org/10.1002/ejsp.8o6. 
Lai, V. T., \& Boroditsky, L. (2013). The immediate and chronic influence of spatiotemporal metaphors on the mental representations of time in English, Mandarin, and Mandarin-English speakers. Frontiers in Psychology, 4, 1-10. http://doi.org/ 10.3389/fpsyg.2013.00142.

Lakoff, G., \& Johnson, M. (1980). Metaphors we live by. Chicago: The University of Chicago Press.

Lee, A., \& Ji, L. J. (2014). Moving away from a bad past and toward a good future: Feelings influence the metaphorical understanding of time.Journal of Experimental Psychology: General, 143(1), 21-26. http://doi.org/10.1037/aoo32233.

Li, H., Bui, Q. Van, \& Cao, Y. (2018). One country, two cultures: Implicit space-time mappings in southern and northern Vietnamese. European Journal of Social Psychology. http://doi.org/10.1002/ejsp.2356.

Loermans, A. C., \& Milfont, T. L. (2018). Time after time: A short-term longitudinal examination of the ego- and time-moving representations. Journal of Research in Personality, 74, 1-5. http://doi.org/10.1016/j.jrp.2017.12.002.

Maass, A. (2009). Culture's two routes to embodiment. European Journal of Social Psychology, 39(7), 129o-1293. http://doi.org/10.1002/ejsp.696.

Margolies, S. O., \& Crawford, L. E. (2008). Event valence and spatial metaphors of time. Cognition \& Emotion, 22(7), 1401-1414. http://doi.org/10.1080/o2699930701810335.

McGlone, M. S., \& Harding, J. L. (1998). Back (or forward?) to the future: The role of perspective in temporal language comprehension. Journal of Experimental Psychology: Learning, Memory, and Cognition, 24(5), 1211-1223. http://doi.org/ 10.1037/o278-7393.24.5.1211.

McGlone, M. S., \& Pfiester, R. A. (2009). Does time fly when you're having fun, or do you? Affect, agency, and embodiment in temporal communication. Journal of Language and Social Psychology, 28(1), 3-31. http://doi.org/10.1177/o261927Xo8325744.

Núñez, R., Cooperrider, K., Doan, D., \& Wassmann, J. (2012). Contours of time: Topographic construals of past, present, and future in the Yupno valley of Papua New Guinea. Cognition, 124, 25-35. http://doi.org/10.1016/j.cognition.2012.03.007.

Núñez, R. E., \& Cooperrider, K. (2013). The tangle of space and time in human cognition. Trends in Cognitive Sciences, 17(5). http://doi.org/10.1016/j.tics.2013.03.008.

Richmond, J., Wilson, C. J., \& Zinken, J. (2012). A feeling for the future: How does agency in time metaphors relate to feelings? European Journal of Social Psychology, 42(7), 813-823. http://doi.org/10.10o2/ejsp.19o6.

Rothe-Wulf, A., Beller, S., \& Bender, A. (2015). Temporal frames of reference in three Germanic languages: Individual consistency, interindividual consensus, and crosslinguistic variability. The Quarterly Journal of Experimental Psychology, 68(5), 917939. http://doi.org/10.1080/17470218.2014.970205. 
Rotteveel, M., Gierholz, A., Koch, G., van Aalst, C., Pinto, Y., Sasiadek, A., \& Wagenmakers, E.-J. (2015). On the automatic link between affect and tendencies to approach and avoid: Chen and Bargh (1999) revisited. Frontiers in Psychology, 6. http://doi .org/10.3389/fpsyg.2015.00335.

Ruscher,J. B. (2011). Moving forward:The effect of spatiotemporal metaphors on perceptions about grief. Social Psychology, 42(3), 225-23o. http://doi.org/10.1027/1864-9335/ aoooo66.

Sinha, C., Sinha, V. D. S., Zinken, J., \& Sampaio, W. (2011). When time is not space: The social and linguistic construction of time intervals and temporal event relations in an Amazonian culture. Language and Cognition, 3(o1), 137-169. http://doi .org/10.1515/langcog.2011.006.

Tversky, B., Kugelmass, S., \& Winter, A. (1991). Cross-cultural and developmental trends in graphic productions. Cognitive Psychology, 23(4), 515-557. http://doi.org/ 10.1016/o010-0285(91)90005-9.

Wu, L., \& Barsalou, L. W. (2009). Perceptual simulation in conceptual combination: Evidence from property generation. Acta Psychologica, 132(2), 173-189. http://doi .org/10.1016/j.actpsy.2009.02.002. 